\title{
Association between the metabolically healthy obese phenotype and the risk of myocardial infarction: results from the Kailuan study
}

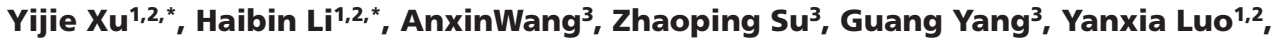 \\ Lixin Tao ${ }^{1,2}$, Shuohua Chen ${ }^{4}$, Shouling Wu${ }^{4}$, Youxin Wang ${ }^{1,2}$ and Xiuhua Guo ${ }^{1,2}$ \\ ${ }^{1}$ Department of Epidemiology and Health Statistics, School of Public Health, Capital Medical University, Beijing, \\ China, ${ }^{2}$ Beijing Municipal Key Laboratory of Clinical Epidemiology, Capital Medical University, Beijing, China, \\ ${ }^{3}$ Department of Neurology, Beijing Tiantan Hospital, Capital Medical University, Beijing, China, and ${ }^{4}$ Department of \\ Cardiology, Kailuan Hospital, North China University of Science and Technology, Tangshan, China \\ $*(\mathrm{Y} \mathrm{Xu}$ and $\mathrm{H} \mathrm{Li}$ contributed equally to this work $)$
}

Correspondence

should be addressed

to $X$ Guo or $Y$ Wang or

$S$ Wu

Email

statguo@ccmu.edu.cn or sdwangyouxin@163.com or drwus|@163.com

\section{Abstract}

Objective: This study aimed to determine if the metabolically healthy obese (MHO) is associated with an increased risk of myocardial infarction (MI) in Chinese population.

Design: The Kailuan study is a community-based prospective cohort study.

Methods: BMI and metabolic syndrome (MetS) were assessed in 91866 participants without a history of MI or stroke. Participants were categorised into six mutually exclusive groups according to the BMI-MetS status: normal weight (BMI: $\leq 18.5$ to $<24.0 \mathrm{~kg} / \mathrm{m}^{2}$ ) without MetS (MH-NW), normal weight with MetS (MUH-NW), overweight (BMI: $\leq 24.0$ to $<28.0 \mathrm{~kg} / \mathrm{m}^{2}$ ) without MetS (MH-OW), overweight with MetS (MUH-OW), obese $\left(\mathrm{BMI} \geq 28.0 \mathrm{~kg} / \mathrm{m}^{2}\right.$ ) without MetS (MHO) and obese with MetS (MUO). The hazard ratio (HR) with $95 \% \mathrm{Cl}$ was calculated for the incidence of $\mathrm{MI}$ using a multivariable Cox model.

Results: A total of 6745 (7.34\%) individuals were classified as MHO. During a median 8-year follow-up, 1167 (1.27\%) participants developed MI. The MHO group had an increased risk of MI (HR: 1.76, 95\% Cl: 1.37-2.25) in comparison with the MH-NW group after adjusting for potential confounding variables. After a similar adjustment, the risk of MI was significantly elevated in the MUH-NW (HR: 1.62, 95\% Cl: 1.28-2.05), MUH-OW (HR: 1.98, 95\% Cl: 1.67-2.35) and MUO group (HR: 2.06, 95\% Cl: 1.70-2.49).

Conclusions: MHO subjects showed a substantially higher risk of $\mathrm{MI}$ in comparison with $\mathrm{MH}-\mathrm{NW}$ subjects. That said, even without measurable metabolic abnormalities, obesity was associated with a higher risk of $\mathrm{MI}$.

\section{Introduction}

As most epidemiological studies have recognised, obesity, measured either by BMI or several other measures of adiposity, is one of the most important modifiable and causal risk factors of myocardial infarction (MI), stroke, cardiovascular diseases (CVDs) and all-cause mortality $(1,2,3)$. Metabolic syndrome (MetS) is characterised by a cluster of conditions, including dyslipidaemia, hyperglycaemia and hypertension (4). Previous meta- (c) 2018 European Society of Endocrinology Printed in Great Britain
Published by Bioscientifica Ltd. 
analyses $(5,6)$ and cohort studies $(7,8,9)$ have shown that MetS significantly increases the risk of CVDs and all-cause mortality. However, a fraction of obese individuals who do not have MetS is considered as metabolically healthy obese (MHO) (10). Correspondingly, a subset of lean individuals who have MetS is referred to as metabolically unhealthy normal weight (MUH-NW).

Previous prospective cohort studies have demonstrated that $\mathrm{MHO}$ individuals are at an increased risk of CVDs and all-cause mortality compared with metabolically healthy normal weight (MH-NW) individuals $(7,9,11,12,13,14,15)$. For example, a recent large-scale study investigating a 30-year follow-up period in 90257 women found that the MHO group had an increased 1.44-fold risk of MI, 1.37-fold risk of stroke and 1.39-fold risk of CVDs (15). On the other hand, some observational studies had reported that the MHO phenotype was not significantly associated with increased risk of CVDs $(16,17)$. The reason for their findings may partially be due to the small sample size in each study and the relatively small fraction of CVD cases over a short-term follow-up. In addition, studies on the association between $\mathrm{MHO}$ and risk of MI have mostly been conducted in the Western population $(8,9)$. Because the pattern and prevalence of obesity and CVDs risk in the Chinese population are different from those in the Western population, it is necessary to perform a large prospective cohort study to further elucidate the impact of MHO phenotype on the risk of MI.

The Kailuan study is a large and ongoing prospective community-based study. Baseline data for BMI, MetS and assessment of MI were collected prospectively to identify the association between the MHO phenotype and risk of MI in the Chinese population. Therefore, the present study aimed to investigate the association between MetS and the risk of MI across BMI categories. We hypothesised that the MHO group had a greater risk of MI than that of the MH-NW group.

\section{Methods}

\section{Study population}

The Kailuan study is a prospective cohort study comprising 101510 participants aged between 18 and 98 years, located in Tangshan, Hebei, China (18). Details regarding the design and methods of the study have been previously published (18). Briefly, beginning in 2006-2007, both questionnaire information and clinical/ laboratory examinations were performed every 2 years.
In the current analysis, we initially excluded 3330 participants with a history of MI or stroke at baseline, and 3801 participants lacking a fasting blood sample. Furthermore, we excluded 834 participants who did not have weight, height or waist circumference measurements as well as 1679 individuals who were underweight $\left(\mathrm{BMI}<18.5 \mathrm{~kg} / \mathrm{m}^{2}\right)$. Therefore, the final sample consisted of 91866 respondents (Supplementary Fig. 1, see section on supplementary data given at the end of this article) The Ethics Committees of both Kailuan General Hospital and Beijing Tian Tan Hospital approved this study, and all participants gave their written informed consent.

\section{Data collection and baseline measurements}

Standardised questionnaires were used to collect sociodemographic and clinical characteristics, including age, sex, educational level (less than high school, high school or above), income ( $<¥ 800 /$ month, $\geq ¥ 800 /$ month), lifestyle, history of diabetes and hypertension, and current antihypertensive, antidiabetic or blood lipidlowering agents. Lifestyle information included smoking status (never, former/current smoker), drinking status (never, former/current drinker), physical activity levels ( $<3$ times/week, $\geq 3$ times/week) and sodium intake $(<10 \mathrm{~g} /$ day, $\geq 10 \mathrm{~g} /$ day). Trained doctors and nurses measured the participants' height, weight, waist circumference (WC) and blood pressure (BP). Height was measured using a fixed stadiometer with a vertical backboard, and weight was measured on a digital scale. BMI was calculated by dividing weight in $\mathrm{kg}$ by height in square metres $\left(\mathrm{kg} / \mathrm{m}^{2}\right)$. WC was measured to the nearest $2.5 \mathrm{~cm}$ above the umbilicus. BP was measured using a mercury sphygmomanometer, and the average of three BP measurements was calculated for systolic and diastolic BP, respectively.

Fasting blood samples were collected in the morning and analysed using an auto-analyser (Hitachi 747; Hitachi). Fasting blood glucose (FBG), triglycerides (TG), total cholesterol (TC), high-density lipoprotein cholesterol (HDL-C), low-density lipoproteins cholesterol (LDL-C), high-sensitivity C-reactive protein (hs-CRP) and serum creatinine were measured using a standardised method (19). Moreover, the estimated glomerular filtration rate (eGFR) was calculated using the Chronic Kidney Disease Epidemiology Collaboration equation (20).

\section{Definition of BMI-MetS groups}

Obesity was defined according to the Working Group on Obesity in China (21) as BMI $\geq 28.0 \mathrm{~kg} / \mathrm{m}^{2}$, overweight 
as $24.0 \leq \mathrm{BMI}<28.0 \mathrm{~kg} / \mathrm{m}^{2}$ and normal weight as $18.5 \leq \mathrm{BMI}<24.0 \mathrm{~kg} / \mathrm{m}^{2}$. MetS was defined as having $\geq 3$ metabolic abnormalities according to the harmonized International Diabetes Federation criteria (4) published in 2009 (Table 1). Participants were divided into six mutually exclusive groups based on their BMI categories (normal weight, overweight and obesity) and the presence or absence of MetS: (1) metabolically healthy normal weight (MH-NW), (2) metabolically healthy overweight (MH-OW), (3) metabolically healthy obese (MHO), (4) metabolically unhealthy normal weight (MUH-NW), (5) metabolically unhealthy overweight (MUH-OW) and (6) metabolically unhealthy obese (MUO) (Table 1).

\section{Assessment of MI}

After the baseline examination, participants were followed up for the first occurrence of MI, including nonfatal and fatal MI. The occurrence of MI was identified from the medical records of all 11 Kailuan hospitals and the Municipal Social Insurance Institution from the baseline examination date until 31 December 2015. Death due to

Table 1 Definition of obesity, metabolic syndrome and metabolically healthy obesity.

- BMI categories were based on the Working Group on Obesity in China:

1. Normal weight: $\mathrm{BMI} \leq 18.5$ to $<24.0 \mathrm{~kg} / \mathrm{m}^{2}$;

2. Overweight: $\mathrm{BMI} \leq 24.0$ to $<28.0 \mathrm{~kg} / \mathrm{m}^{2}$;

3. Obesity: $B M I \geq 28.0 \mathrm{~kg} / \mathrm{m}^{2}$;

- Metabolic syndrome (MetS) was defined as $\geq 3$ of following components based on the harmonized International Diabetes Federation criteria:

1. Systolic blood pressure $\geq 130$ and/or diastolic blood pressure $\geq 85 \mathrm{mmHg}$ and/or use of antihypertensive medication and/or self-reported history of hypertension;

2. Fasting blood glucose $\geq 5.6 \mathrm{mmol} / \mathrm{L}(100 \mathrm{mg} / \mathrm{dL})$ and/ or current use of antidiabetic medication and/or selfreported history of diabetes;

3. Triglycerides $\geq 1.7 \mathrm{mmol} / \mathrm{L}(150 \mathrm{mg} / \mathrm{dL})$ or current use of lipid-lowering medication;

4. High-density lipoprotein cholesterol $<1.0 \mathrm{mmol} / \mathrm{L}(40 \mathrm{mg} /$ $\mathrm{dL}$ ) for men and $<1.3 \mathrm{mmol} / \mathrm{L}(50 \mathrm{mg} / \mathrm{dL})$ for women;

5. Waist circumference $\geq 85 \mathrm{~cm}$ for $\operatorname{man}$ and $\geq 80 \mathrm{~cm}$ for women;

- BMI-MetS groups:

1. Metabolically healthy normal weight (MH-NW): normal weight without MetS;

2. Metabolically healthy overweight $(\mathrm{MH}-\mathrm{OW})$ : overweight without Mets;

3. Metabolically healthy obese (MHO): obese without MetS;

4. Metabolically unhealthy normal weight (MUH-NW): normal weight with MetS;

5. Metabolically unhealthy overweight (MUH-OW): overweight with MetS;

6. Metabolically unhealthy obese (MUO): obese with MetS;
MI was verified from death certificates from the provincial vital statistic offices. The diagnostic criteria of MI were consistent across all 11 hospitals collectively based on chest pain symptoms, electrocardiographic changes and cardiac enzyme levels.

\section{Statistical analysis}

All statistical analyses were performed using the SAS software version 9.4 (SAS Institute Inc), and a two-sided $P$ value of $<0.05$ was considered statistically significant. Characteristics of participants were summarised according to the BMI-MetS categories. Continuous variables are expressed as mean \pm standard deviation and categorical variables are presented as frequencies and percentages. Trend association was assessed by assigning a median BMI value to each categories of BMI in both the metabolically healthy and metabolically unhealthy groups and tested using linear regression or logistic regression, as appropriate.

Person-years were calculated from the baseline examination date until the date of MI diagnosis, death, lost follow-up or the end of follow-up (31 December 2015), whichever occurred first. The time to occurrence of MI was first estimated using the Kaplan-Meier method, and the BMI-MetS group differences were tested using the log-rank test. The Šidák method was applied to obtain a multiple-comparison adjustment of $P$ values for the paired comparison. Hazard ratio (HR) and corresponding 95\% CI were estimated using Cox proportional hazards models. The proportional hazards assumption was checked by plotting the Schoenfeld residuals (22) and no violation was found. Four Cox models were applied: model 1 was unadjusted; model 2 was adjusted for age and sex; model 3 was adjusted for age, sex, educational level, income, smoking, drinking, physical activity and sodium intake and model 4 was adjusted for covariates in model 3 plus LDL-C, hs-CRP and eGFR. We chose these sociodemographic factors and traditional CVDs risk factors as adjusted covariates, which were proved to be associated with CVDs in previous report of the Kailuan study $(18,19)$.

Multivariable adjusted model was stratified by the following potential effect modifiers: sex (male or female), age ( $<65$ or $\geq 65$ years), smoking status (never or former/current smoker), drinking status (never or former/current drinker) and physical activity (yes or no). The multiplicative interaction terms between these subgroups and BMI-MetS status were added to the fully adjusted model, and model with and without multiplicative interaction terms were compared using 


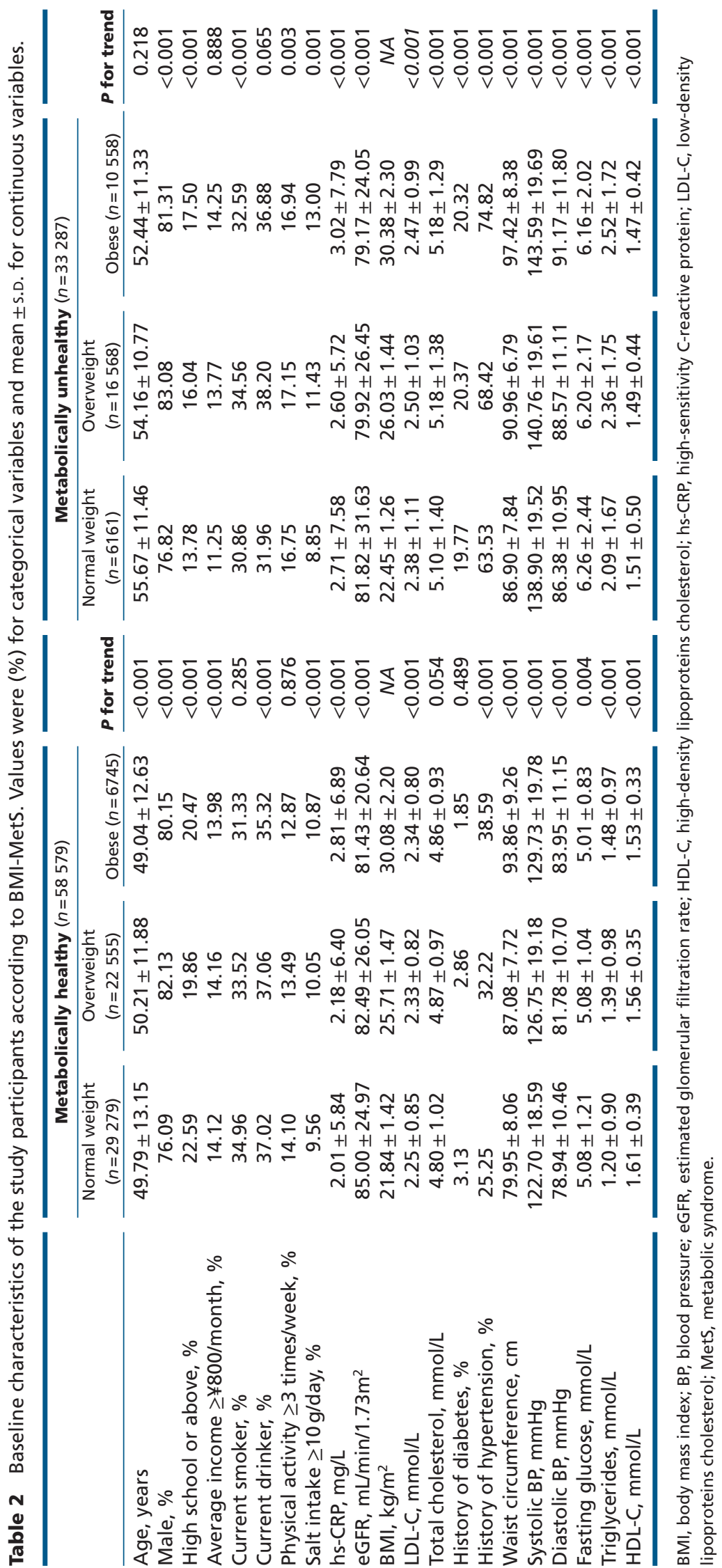


the likelihood ratio test. In addition, several sensitivity analyses were performed to confirm the robustness of our findings, including (1) considering non-MI death as a competing risk event rather than a censoring event, where Fine-Gray competing risk model was applied to address this issue (23); (2) modifying the definition of MetS defined as $\geq 2$ among the four components (Table 1, excludes the WC criterion) (13) and (3) using the World Health Organization criteria (24) to define normal weight $\left(18.5 \leq \mathrm{BMI}<25.0 \mathrm{~kg} / \mathrm{m}^{2}\right)$, overweight $\left(25.0 \leq \mathrm{BMI}<30.0 \mathrm{~kg} / \mathrm{m}^{2}\right)$ and obese $\left(\mathrm{BMI} \geq 30.0 \mathrm{~kg} / \mathrm{m}^{2}\right)$.

\section{Results}

Table 2 shows the baseline characteristics of the participants according to BMI-MetS categories. In total, 33287 (33.26\%) participants were classified as metabolically unhealthy and 58579 (63.77\%) as metabolically healthy. Overall, $18.83 \%$ of participants $(n=17303)$ were obese. The MHO phenotype represented $7.34 \%(n=6745)$ of the total analytic sample and $38.98 \%$ of the obese population. TG, LDL-C, FBG, WC and BP were higher in the metabolically unhealthy group. There was no difference in the proportions of physically active individuals in the MH-NW, MH-OW and MHO groups.

Over a median of 8.09 years (interquartile range: 7.82-8.24 years) of follow-up, 1167 (1.27\%) incident MI cases were documented. The crude incidence rate per 1000 person-years of MI was 1.12 in the MH-NW group, 1.09 in the MH-OW group, 1.71 in the MHO group, 2.11 in the MUH-NW group, 2.46 in the MUH-OW group and 2.42 in the MUO group. Figure 1 shows the cumulative hazard for MI stratified by the BMI-MetS group (log-rank test, $P<0.001)$. These curves significantly differed from each other except for MH-NW vs MH-OW $(P=0.780)$, MHO vs MUH-NW $(P=0.973)$, MUH-NW vs MUO $(P=0.197)$ and MUH-OW vs MUO $(P=0.098)$.

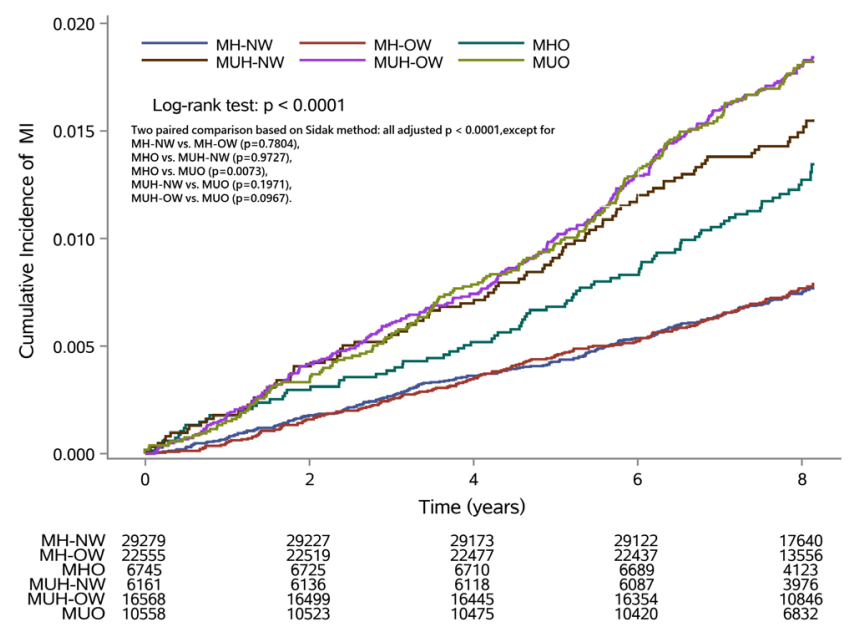

Figure 1

Cumulative incidences of myocardial infarction (MI) according to the combined BMI category (normal weight, overweight, obese) and metabolic syndrome (absence or presence). A full colour version of this figure is available at https://doi. org/10.1530/EJE-18-0356.

Table 3 shows the association between the BMI-MetS group and risk of MI. Overall, the adjusted HR (model 4 in Table 3) for the incidence of MI in MHO individuals (MH-NW group as the reference) was 1.76 (95\% CI: 1.37-2.25). The adjusted HR for the incidence of MI in the MUH-NW (HR: 1.62, 95\% CI: 1.28-2.05) and MUO (HR: 2.06, 95\% CI: 1.70-2.49) group was also significantly higher than that in the MH-NW group.

Associations between the BMI-MetS group and risk of MI did not significantly differ by sex, age group, smoking status, drinking status or physical activity (all $P>0.05$ for heterogeneity, Table 4), and sensitivity analyses showed similar results (Supplementary Table 1). The association between MHO and risk of MI was mildly attenuated after adjusting for competing risk of non-MI death (HR:

Table 3 Association of BMI-MetS at baseline and risk of myocardial infarction. Data was reported as hazard ratio and $95 \%$ confidence intervals.

\begin{tabular}{|c|c|c|}
\hline & MI, $n$ & Incidence rate\# \\
\hline Metabolically healthy normal weight & 246 & 1.05 \\
\hline Metabolically healthy overweight & 197 & 1.09 \\
\hline Metabolically healthy obese & 92 & 1.71 \\
\hline Metabolically unhealthy normal weight & 104 & 2.11 \\
\hline Metabolically unhealthy overweight & 325 & 2.46 \\
\hline Metabolically unhealthy obese & 204 & 2.42 \\
\hline
\end{tabular}

\begin{tabular}{|c|c|c|c|}
\hline Model $1^{+}$ & Model $2^{\ddagger}$ & Model $3^{\S}$ & Model 4" \\
\hline$\overline{1.00 \text { (Reference) }}$ & $\overline{1.00 \text { (Reference) }}$ & $\overline{1.00 \text { (Reference) }}$ & 1.00 \\
\hline & 1.10 & 1.09 & 1 \\
\hline 1.71( & 1.86 & 1.79 & 1.7 \\
\hline 2.04( & 1.72( & 1.62( & 1.62 \\
\hline $2.32(1.97-2.75)$ & $2.08(1.76-2.46)$ & $2.03(1.71-2.40)$ & $1.98(1.67-2.3$ \\
\hline $2.19(1.82-2.64)$ & $2.17(1.80-2.62)$ & $2.13(1.76-2.58)$ & $2.06(1.70-2.49$ \\
\hline
\end{tabular}

\#Per 1000 person-years. ${ }^{\dagger}$ Model 1 was unadjusted. ${ }^{\ddagger}$ Model 2 was adjusted for age and sex. ${ }^{\S}$ Model 3 was adjusted for age, sex, educational level, income, smoking, drinking, physical activity and sodium intake. "Model 4 was adjusted for in Model 3 plus LDL cholesterol, high-sensitive C-reactive protein and glomerular filtration rate (eGFR). MI, myocardial infarction 
1.67, 95\% CI: 1.30-2.14). Excluding the WC criterion from the definition of MetS demonstrated a somewhat stronger association between the MHO group and MI (HR: 1.84, 95\% CI: 1.42-2.38). Finally, when obesity was defined as $\mathrm{BMI} \geq 30 \mathrm{~kg} / \mathrm{m}^{2}$, the HR for MI was somewhat stronger in all of BMI-MetS group except the MUH-NW group. The MHO group was associated with a 2.19-fold (95\% CI: 1.58-3.03) risk of MI compared with that of the MH-NW group.

\section{Discussion}

In this prospective cohort study, we examined the combined effects of BMI-MetS on the risk of MI over an 8-year follow-up. The main finding of this study was that $\mathrm{MHO}$ subjects had roughly a 1.8-fold higher risk of incidence of $\mathrm{MI}$ in comparison with their nonobese counterparts (MH-NW).

BMI, a measure of general adiposity, is an established causal risk factor for CVDs and type 2 diabetes $(7,25)$. Based on the Kailuan study, our previous studies demonstrated that BMI, WC, waist-to-hip ratio and waist-to-height ratio were also associated with $\mathrm{MI}$, ischaemic stroke and diabetes $(18,19)$. The current study further elucidated that obesity had a strong positive association with the risk of MI regardless of having MetS, suggesting that the management of excess weight seemed to be necessary for all individuals. Findings from the community-based Uppsala Longitudinal Study of Adult Men also supported our results (7).

There remains considerable debate regarding the criteria for the definition of the MHO phenotype (26). Proposed by Wildman (27), Aguilar-Salinas (28), Meigs (29, 30), Karelis (31) and the National Cholesterol Education Program-Adult Treatment Panel III (ATP-III) (32), these criteria were commonly used to assess metabolic status. Thus, the prevalence of the MHO phenotype may vary widely due to the different definitions and diversified study population characteristics $(33,34)$. Using the International Diabetes Federation consensus definition, the prevalence of the MHO phenotype in the present study was $7.34 \%$ in Chinese adults. Our results were consistent with those of a recent meta-analysis, reporting a the prevalence rate of $7.27 \%$ (95\% CI: 5.93-8.90\%) for $\mathrm{MHO}$ (33). The prevalence of $\mathrm{MHO}$ in the Chinese population was reported to range from 4 to $20 \%(35,36,37,38)$. However, some studies had relatively small sample sizes $(n<5000)(35,36)$. Notably, the prevalence of the MHO phenotype was related to metabolically healthy criteria, sample size and study population characteristics (33).
Therefore, it is necessary to formulate a comprehensive definition of 'metabolically healthy' and identify highrisk groups in order to prevent CVDs.

Numerous prospective cohort studies have previously investigated the association between MHO phenotype and CVDs risk as a composite outcome $(7,9,11,12,13$, $14,15,16,25,34,39$, 40), whereas specific outcomes, such as MI were limited. Our results were also consistent with those of recent meta-analyses showing that $\mathrm{MHO}$ individuals were at increased risk for cardiovascular events over a long-term follow-up (41). Similar to our findings, the Copenhagen General Population Study of 71527 individuals identified $\mathrm{MHO}$ as being associated with a higher risk of MI (HR: 1.80, 95\% CI: 1.34-2.63) compared with MH-NW subjects (9). In the San Antonio Heart Study, MUH-NW and MHO individuals were associated with an increased risk of incident CVDs (HR: 2.9, 95\% CI: 1.3-6.4 and HR: 3.9, 95\% CI: 1.9-7.8, respectively) (25). Interestingly, in the Nord-Trøndelag Health Study, where 61299 participants were followed up with a median of 12.2 years, Morkedal et al. found that MHO individuals did not have an increased risk of MI in comparison with MH-NW subjects (HR: 1.10, 95\% CI: 0.90-1.40) (8). We considered such inconsistencies between findings of the former study and our study may be due to differences in the definition of obesity and the population characteristics. There are different BMI cut-off values for obesity. The estimated association of $\mathrm{MHO}$ and MI may vary according to the different BMI cut-off values for obesity in the NordTrøndelag Health Study. In our sensitivity analysis, when using $\mathrm{BMI} \geq 30 \mathrm{~kg} / \mathrm{m}^{2}$ for obesity, the effect estimate of MI was significantly larger than that estimated in the NordTrøndelag Health Study (HR: 2.19 vs 1.10). In fact, Asians have a higher amount of visceral fat at the same level of BMI compared to Caucasians (42). Abdominal obesity has been linked to increased risks of MetS and type 2 diabetes $(18,19)$; therefore, Chinese individuals may have a higher risk for CVDs at the same level of obesity. This may explain why the current study population was at higher risk of MI as compared that in Nord-Trøndelag Health Study.

The association between $\mathrm{MHO}$ and cardiovascular outcomes in the elderly remain controversial $(16,43)$. In the subpopulation aged $\geq 65$ years, we did not find a high risk of $\mathrm{MI}$ in $\mathrm{MHO}$ individuals compared with the NM-NW group (HR: 1.04, 95\% CI: 0.60-1.80). Our findings were consistent with the finding those of the Rotterdam Study (16), which study also reported that MHO did not confer a higher cardiovascular risk in 5314 individuals aged $\geq 55$ years (16). The different impacts of $\mathrm{MHO}$ on cardiovascular risk may be due to the differences 


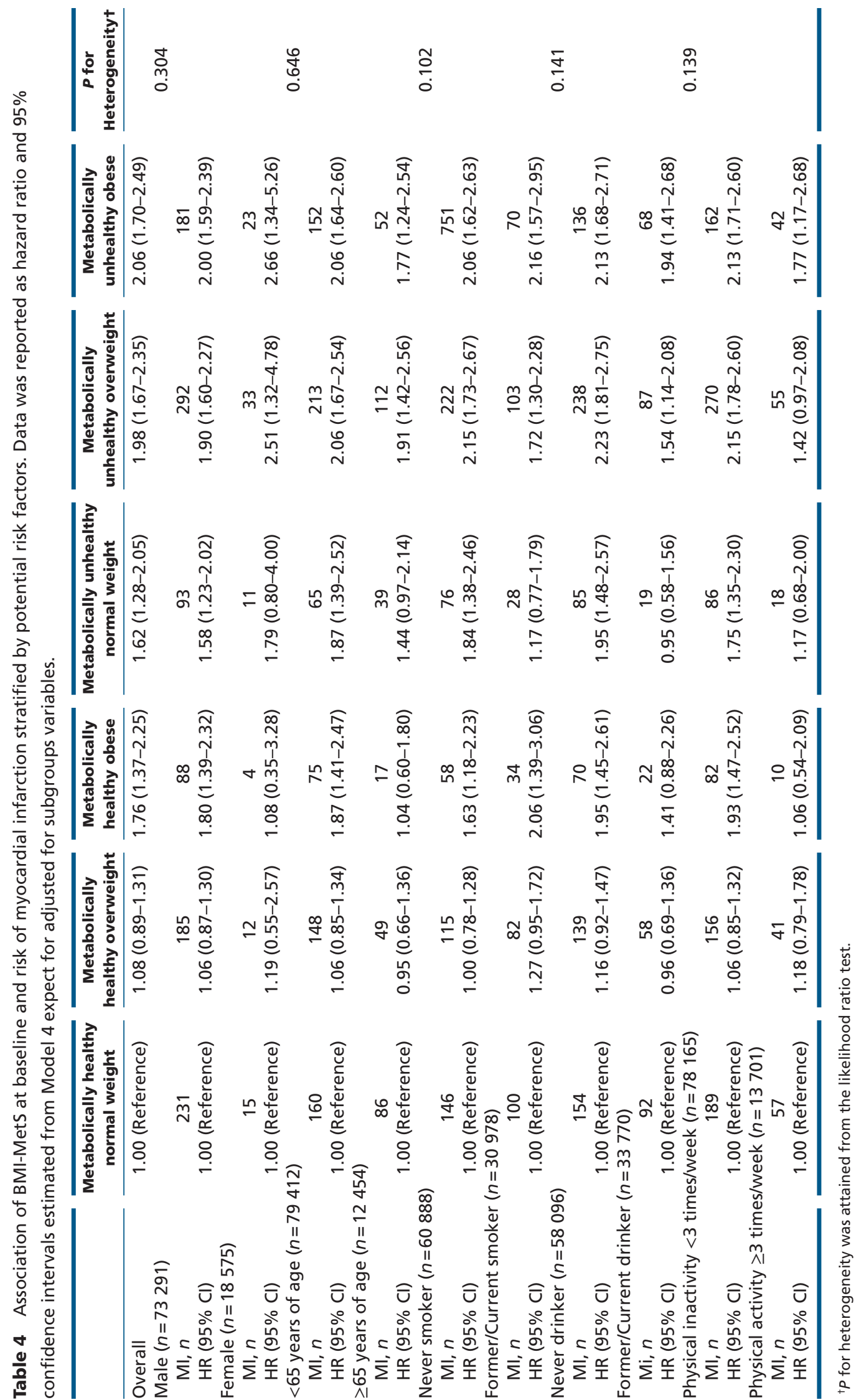


in body weight, body composition and health behaviour between the young and the elderly (44). The interaction term was not statistically significant, either. In addition, we did not find any potential effect modifiers, such as sex, smoking status or physical activity. However, caution should be taken when interpreting the results of the subgroup analysis due to the small numbers of MI events in certain subgroups.

Our study has several limitations. First, MHO is an unstable state (14), and we did not assess the impact of the transition from MHO to MUO on MI prospectively. The recent Multi-Ethnic Study of Atherosclerosis Study found that almost half of MHO subjects developed MUO (45). Notably, subjects with unstable MHO had a higher risk of CVDs than that of subjects with stable MHO or healthy normal weight (45). Second, although we cautiously adjusted for potential confounders, we could not adjust for some residual or unmeasured confounding factors, e.g., social deprivation and depressive symptoms, as previous study reported an increased odds of depressive symptoms in the MHO group (46). Third, participants in the present study were recruited from the Kailuan community located in the city of Tangshan, Hebei Province, China. This study cohort was not nationally representative, and the association between $\mathrm{MHO}$ and risk of $\mathrm{MI}$ in this population may not be directly generalised to other populations. Our study also has several strengths, including the prospective cohort design, large sample size in a fixed functional community in China and a standardised evaluation of directly measured metabolic markers and body sizes. Moreover, we affirmed the combined effect of obesity and MetS on the risk of MI. Our findings suggest that MHO is not a low-risk state. In addition, our future work will focus on the association between the transition from metabolically healthy to unhealthy phenotypes and the risk of CVDs in this population.

\section{Conclusions}

In conclusion, our study showed that MHO individuals had a substantially increased risk of incident $\mathrm{MI}$ in comparison with MH-NW individuals in a large Chinese community, indicating that all obese individuals, even those without MetS should be encouraged to lower their weight to reduce the risk of MI.

\section{Supplementary data}

This is linked to the online version of the paper at https://doi.org/10.1530/ EJE-18-0356.
Declaration of interest

The authors declare that there is no conflict of interest that could be perceived as prejudicing the impartiality of this study.

\section{Funding}

This work was supported by the Program of Natural Science Fund of China (Serial Number: 81530087, 81673247, 81872708).

\section{Authors contribution statement}

$H \mathrm{~L}$ and $\mathrm{Y} X$ applied data and wrote the manuscript. A $W$ contributed to introduction and discussion. Z S, Y W, L T, S C contributed to the discussion. $S \mathrm{~W}, \mathrm{Y} X$ and $X \mathrm{G}$ contributed to the discussion and reviewed and edited the manuscript. All authors read and approved the final manuscript.

\section{Acknowledgements}

The authors thank all the staff and participants of the Kailuan study for their invaluable contributions. Additionally, they would like to thank Prof. Wu of the Temple University of the United States for polishing the language.

\section{References}

1 Hagg S, Fall T, Ploner A, Magi R, Fischer K, Draisma HHM, Kals M, de Vries PS, Dehghan A, Willems SM et al. Adiposity as a cause of cardiovascular disease: a Mendelian randomization study. International Journal of Epidemiology 201544 578-586. (https://doi. org/10.1093/ije/dyv094)

2 Twig G, Yaniv G, Levine H, Leiba A, Goldberger N, Derazne E, Ben-Ami Shor D, Tzur D, Afek A, Shamiss A et al. Body-mass index in 2.3 million adolescents and cardiovascular death in adulthood. New England Journal of Medicine 2016374 2430-2440. (https://doi. org/10.1056/NEJMoa1503840)

3 Afzal S, Tybjaerg-Hansen A, Jensen GB \& Nordestgaard BG. Change in body mass index associated with lowest mortality in Denmark, 1976-2013. JAMA 2016315 1989-1996. (https://doi.org/10.1001/ jama.2016.4666)

4 Alberti KG, Eckel RH, Grundy SM, Zimmet PZ, Cleeman JI, Donato KA, Fruchart JC, James WP, Loria CM, Smith SC Jr et al. Harmonizing the metabolic syndrome: a joint interim statement of the International Diabetes Federation Task Force on Epidemiology and Prevention; National Heart, Lung, and Blood Institute; American Heart Association; World Heart Federation; International Atherosclerosis Society; and International Association for the Study of Obesity. Circulation 2009120 1640-1645. (https://doi. org/10.1161/CIRCULATIONAHA.109.192644)

5 Gami AS, Witt BJ, Howard DE, Erwin PJ, Gami LA, Somers VK \& Montori VM. Metabolic syndrome and risk of incident cardiovascular events and death: a systematic review and meta-analysis of longitudinal studies. Journal of the American College of Cardiology 2007 49 403-414. (https://doi.org/10.1016/j.jacc.2006.09.032)

6 Mottillo S, Filion KB, Genest J, Joseph L, Pilote L, Poirier P, Rinfret S, Schiffrin EL \& Eisenberg MJ. The metabolic syndrome and cardiovascular risk a systematic review and meta-analysis. Journal of the American College of Cardiology 201056 1113-1132. (https://doi. org/10.1016/j.jacc.2010.05.034)

7 Arnlov J, Ingelsson E, Sundstrom J \& Lind L. Impact of body mass index and the metabolic syndrome on the risk of cardiovascular disease and death in middle-aged men. Circulation 2010121 230-236. (https://doi.org/10.1161/CIRCULATIONAHA.109.887521) 
8 Morkedal B, Vatten LJ, Romundstad PR, Laugsand LE \& Janszky I. Risk of myocardial infarction and heart failure among metabolically healthy but obese individuals: HUNT (Nord-Trøndelag Health Study), Norway. Journal of the American College of Cardiology 201463 1071-1078. (https://doi.org/10.1016/j.jacc.2013.11.035)

9 Thomsen M \& Nordestgaard BG. Myocardial infarction and ischemic heart disease in overweight and obesity with and without metabolic syndrome. JAMA Internal Medicine 2014174 15-22. (https://doi. org/10.1001/jamainternmed.2013.10522)

10 Tomiyama AJ, Hunger JM, Nguyen-Cuu J \& Wells C. Weight and cardiometabolic health: new perspectives. International Journal of Obesity (Lond) 201640 1331. (https://doi.org/10.1038/ijo.2016.83)

11 Caleyachetty R, Thomas GN, Toulis KA, Mohammed N, Gokhale KM, Balachandran K \& Nirantharakumar K. Metabolically healthy obese and incident cardiovascular disease events among 3.5 million men and women. Journal of the American College of Cardiology $2017 \mathbf{7 0}$ 1429-1437. (https://doi.org/10.1016/j.jacc.2017.07.763)

12 Hinnouho GM, Czernichow S, Dugravot A, Nabi H, Brunner EJ, Kivimaki M \& Singh-Manoux A. Metabolically healthy obesity and the risk of cardiovascular disease and type 2 diabetes: the Whitehall II cohort study. European Heart Journal 201536 551-559. (https://doi. org/10.1093/eurheartj/ehu123)

13 Lassale C, Tzoulaki I, Moons KGM, Sweeting M, Boer J, Johnson L, Huerta JM, Agnoli C, Freisling H, Weiderpass E et al. Separate and combined associations of obesity and metabolic health with coronary heart disease: a pan-European case-cohort analysis. European Heart Journal 201839 397-406. (https://doi.org/10.1093/ eurheartj/ehx448)

14 Kuk JL \& Ardern CI. Are metabolically normal but obese individuals at lower risk for all-cause mortality? Diabetes Care 200932 2297-2299. (https://doi.org/10.2337/dc09-0574)

15 Eckel N, Li Y, Kuxhaus O, Stefan N, Hu FB \& Schulze MB. Transition from metabolic healthy to unhealthy phenotypes and association with cardiovascular disease risk across BMI categories in 90257 women (the Nurses' Health Study). Lancet Diabetes Endocrinology 2018. (https://doi.org/10.1016/S2213-8587(18)30137-2)

16 Dhana K, Koolhaas CM, van Rossum EF, Ikram MA, Hofman A, Kavousi M \& Franco OH. Metabolically healthy obesity and the risk of cardiovascular disease in the elderly population. PLOS ONE 2016 11 e0154273. (https://doi.org/10.1371/journal.pone.0154273)

17 Hamer M \& Stamatakis E. Metabolically healthy obesity and risk of all-cause and cardiovascular disease mortality. Journal of Clinical Endocrinology and Metabolism 201297 2482-2488. (https://doi. org/10.1210/jc.2011-3475)

18 Wang A, Wu J, Zhou Y, Guo X, Luo Y, Wu S \& Zhao X. Measures of adiposity and risk of stroke in China: a result from the Kailuan study. PLOS ONE 2013 8. (https://doi.org/10.1371/journal. pone.0061665)

19 Wang A, Li Z, Zhou Y, Wang C, Luo Y, Liu X, Guo X, Wu S \& Zhao X. Hypertriglyceridemic waist phenotype and risk of cardiovascular diseases in China: results from the Kailuan Study. International Journal of Cardiology 2014174 106-109. (https://doi.org/10.1016/j. ijcard.2014.03.177)

20 Levey AS, Stevens LA, Schmid CH, Zhang YP, Castro AF, Feldman HI, Kusek JW, Eggers P, Van Lente F, Greene T et al. A new equation to estimate glomerular filtration rate. Annals of Internal Medicine 2009 150 604-612. (https://doi.org/10.7326/0003-4819-150-9-20090505000006)

21 Chen C, Lu FC \& Department of Disease Control Ministry of Health PRC. The guidelines for prevention and control of overweight and obesity in Chinese adults. Biomedical and Environmental Sciences 2004 17 1-36.

22 Winnett A \& Sasieni P. Miscellanea. A note on scaled Schoenfeld residuals for the proportional hazards model. Biometrika $2001 \mathbf{8 8}$ 565-571. (https://doi.org/10.1093/biomet/88.2.565)
23 Austin PC, Lee DS \& Fine JP. Introduction to the analysis of survival data in the presence of competing risks. Circulation $2016 \mathbf{1 3 3}$ 601-609. (https://doi.org/10.1161/CIRCULATIONAHA.115.017719)

24 Obesity: preventing and managing the global epidemic. Report of a WHO consultation. World Health Organization Technical Report Series 2000894 i-xii, 1-253.

25 Aung K, Lorenzo C, Hinojosa MA \& Haffner SM. Risk of developing diabetes and cardiovascular disease in metabolically unhealthy normal-weight and metabolically healthy obese individuals. Journal of Clinical Endocrinology and Metabolism 201499 462-468. (https:// doi.org/10.1210/jc.2013-2832)

26 Phillips CM. Metabolically healthy obesity: definitions, determinants and clinical implications. Reviews in Endocrine and Metabolic Disorders 201314 219-227. (https://doi.org/10.1007/s11154-013-9252-x)

27 Wildman RP, Muntner P, Reynolds K, McGinn AP, Rajpathak S, Wylie-Rosett J \& Sowers MR. The obese without cardiometabolic risk factor clustering and the normal weight with cardiometabolic risk factor clustering - Prevalence and correlates of 2 phenotypes among the US population (NHANES 1999-2004). Archives of Internal Medicine 2008168 1617-1624. (https://doi.org/10.1001/archinte.168.15.1617)

28 Aguilar-Salinas CA, Garcia EG, Robles L, Riano D, Ruiz-Gomez DG, Garcia-Ulloa AC, Melgarejo MA, Zamora M, Guillen-Pineda LE, Mehta R et al. High adiponectin concentrations are associated with the metabolically healthy obese phenotype. Journal of Clinical Endocrinology and Metabolism 200893 4075-4079. (https://doi. org/10.1210/jc.2007-2724)

29 Meigs JB, Wilson PW, Fox CS, Vasan RS, Nathan DM, Sullivan LM $\&$ D'Agostino RB. Body mass index, metabolic syndrome, and risk of type 2 diabetes or cardiovascular disease. Journal of Clinical Endocrinology and Metabolism 200691 2906-2912. (https://doi. org/10.1210/jc.2006-0594)

30 Messier V, Karelis AD, Prud'homme D, Primeau V, Brochu M \& Rabasa-Lhoret R. Identifying metabolically healthy but obese individuals in sedentary postmenopausal women. Obesity 201018 911-917. (https://doi.org/10.1038/oby.2009.364)

31 Karelis A, Brochu M \& Rabasa-Lhoret R. Can we identify metabolically healthy but obese individuals (MHO)? Diabetes and Metabolism 200430 569-572. (https://doi.org/10.1016/S12623636(07)70156-8)

32 Grundy SM, Becker D, Clark LT, Cooper RS, Denke MA, Howard WJ, Hunninghake DB, Illingworth R, Luepker RV, McBride P. Third report of the National Cholesterol Education Program (NCEP) expert panel on detection, evaluation, and treatment of high blood cholesterol in adults (adult treatment panel III) final report. Circulation 2002106 3143-3421. (https://doi.org/10.1161/circ.106.25.3143)

33 Wang B, Zhuang R, Luo X, Yin L, Pang C, Feng T, You H, Zhai Y, Ren Y, Zhang L et al. Prevalence of metabolically healthy obese and metabolically obese but normal weight in adults worldwide: A metaanalysis. Hormone and Metabolic Research 201547 839-845. (https:// doi.org/10.1055/s-0035-1559767)

34 Ogorodnikova AD, Kim M, McGinn AP, Muntner P, Khan U \& Wildman RP. Incident cardiovascular disease events in metabolically benign obese individuals. Obesity 201220 651-659. (https://doi. org/10.1038/oby.2011.243)

35 Hwang LC, Bai CH, Sun CA \& Chen CJ. Prevalence of metabolically healthy obesity and its impacts on incidences of hypertension, diabetes and the metabolic syndrome in Taiwan. Asia Pacific Journal of Clinical Nutrition 201221 227-233.

36 Zhang N, Chen Y, Guo X, Sun G \& Sun Y. The prevalence and predictors of metabolically healthy obesity in obese rural population of China: a cross-sectional study. Psychology, Health and Medicine 2017 22 303-309. (https://doi.org/10.1080/13548506.2016.1220596)

37 Chen YT, Zhang NJ, Sun GZ, Guo XF, Yu SS, Yang HM, Zheng LQ \& Sun YX. Metabolically healthy obesity also has risk for hyperuricemia among Chinese general population: A cross-sectional study. 
Obesity Research and Clinical Practice 201610 S84-S95. (https://doi. org/10.1016/j.orcp.2016.03.008)

38 Wang BY, Zhang M, Wang S, Wang CJ, Wang JJ, Li LL, Zhang L, Ren YC, Han CY, Zhao Y et al. Dynamic status of metabolically healthy overweight/obesity and metabolically unhealthy and normal weight and the risk of type 2 diabetes mellitus: A cohort study of a rural adult Chinese population. Obesity Research and Clinical Practice 201812 61-71. (https://doi.org/10.1016/j. orcp.2017.10.005)

39 Mirzaei B, Abdi H, Serahati S, Barzin M, Niroomand M, Azizi F \& Hosseinpanah F. Cardiovascular risk in different obesity phenotypes over a decade follow-up: Tehran Lipid and Glucose Study. Atherosclerosis 2017258 65-71. (https://doi.org/10.1016/j. atherosclerosis.2017.02.002)

40 Kwon BJ, Kim DW, Her SH, Kim DB, Jang SW, Cho EJ, Ihm SH, Kim HY, Youn HJ, Seung KB et al. Metabolically obese status with normal weight is associated with both the prevalence and severity of angiographic coronary artery disease. Metabolism: Clinical and Experimental 201362 952-960. (https://doi.org/10.1016/j. metabol.2013.01.006)

41 Kramer CK, Zinman B \& Retnakaran R. Are metabolically healthy overweight and obesity benign conditions? A systematic review and meta-analysis. Annals of Internal Medicine 2013159 758-769. (https:// doi.org/10.7326/0003-4819-159-11-201312030-00008)
42 Consultation WHOE. Appropriate body-mass index for Asian populations and its implications for policy and intervention strategies. Lancet 2004363 157-163. (https://doi.org/10.1016/S01406736(03) 15268-3)

43 Song YQ, Manson JE, Meigs JB, Ridker PM, Buring JE \& Liu SM. Comparison of usefulness of body mass index versus metabolic risk factors in predicting 10-year risk of cardiovascular events in women. American Journal of Cardiology 2007100 1654-1658. (https://doi. org/10.1016/j.amjcard.2007.06.073)

44 Villareal DT, Apovian CM, Kushner RF, Klein S, American Society for Nutrition \& NAASO, The Obesity Society. Obesity in older adults: technical review and position statement of the American Society for Nutrition and NAASO, the Obesity Society. American Journal of Clinical Nutrition 200582 923-934. (https://doi.org/10.1093/ ajcn/82.5.923)

45 Mongraw-Chaffin M, Foster MC, Anderson CAM, Burke GL, Haq N, Kalyani RR, Ouyang P, Sibley CT, Tracy R, Woodward M et al. Metabolically healthy obesity, transition to metabolic syndrome, and cardiovascular risk. Journal of the American College of Cardiology 2018 71 1857-1865. (https://doi.org/10.1016/j.jacc.2018.02.055)

46 Jokela M, Hamer M, Singh-Manoux A, Batty GD \& Kivimaki M. Association of metabolically healthy obesity with depressive symptoms: pooled analysis of eight studies. Molecular Psychiatry 2014 19 910-914. (https://doi.org/10.1038/mp.2013.162)

Received 24 April 2018

Revised version received 26 August 2018

Accepted 6 September 2018 